Psychological Medicine

cambridge.org/psm

\section{Editorial}

Cite this article: Jauhar S, Laws KR, McKenna PJ (2019). CBT for schizophrenia: a critical viewpoint. Psychological Medicine 49, 1233-1236. https://doi.org/10.1017/ S0033291718004166

Received: 18 November 2018 Revised: 29 November 2018 Accepted: 19 December 2018 First published online: 13 February 2019

Author for correspondence:

S. Jauhar, E-mail: sameer.jauhar@kcl.ac.uk

\title{
CBT for schizophrenia: a critical viewpoint
}

\section{S. Jauhar ${ }^{1}$, K. R. Laws² and P. J. McKenna ${ }^{3}$}

${ }^{1}$ Department of Psychological Medicine, Institute of Psychiatry, Psychology and Neuroscience, London, UK; ${ }^{2}$ School of Life and Medical Sciences, University of Hertfordshire, Hatfield, UK and ${ }^{3}$ FIDMAG Research Foundation, Barcelona and CIBERSAM, Spain

In England and Wales, cognitive behavioral therapy (cognitive therapy, CBT) has been part of official treatment guidelines for schizophrenia since 2002 (NICE, 2009, p. 212). The 2014 NICE guideline (NICE, 2014), which is based on the same meta-analytic evidence as its predecessor in 2009 , recommends that it be offered to all people with schizophrenia, including first-episode patients and those with established illness, and to patients who are actively symptomatic and in remission. Similar recommendations are to be found in the Scottish SIGN guideline (SIGN, 2013) as well as those of several other countries (Rathod et al., 2010).

In 2012, however, the Cochrane collaboration sounded a discordant note, concluding that ' $\mathrm{t}$ ]rial-based evidence suggests no clear and convincing advantage for cognitive behavioural therapy over other-and sometimes much less sophisticated-therapies for people with schizophrenia' (Jones et al., 2012). A further challenge came from a 2014 meta-analysis carried out by ourselves (Jauhar et al., 2014), which found end of treatment effect sizes (ESs) that were uniformly in the small range [overall symptoms: 0.33 (95\% CI 0.19-0.47), 34 studies; positive symptoms: 0.25 (95\% CI 0.13-0.37), 33 studies; negative symptoms: 0.13 (95\% CI 0.01-0.25), 34 studies] (note these and all further ESs are shown as a positive sign favoring CBT). A 2018 network meta-analysis of various psychological interventions to reduce positive symptoms in schizophrenia (Bighelli et al., 2018), carried out on a rather different dataset of 27 studies than us, again found pooled ESs for CBT in the small range, though this time at the upper end of this [ $v$. treatment as usual (TAU): 0.30 (95\% CI 0.14-0.45), 18 trials; $v$. inactive control interventions: 0.29 (95\% CI 0.03-0.55), seven trials] (Bighelli et al., 2018). Most recently, a 2018 update of the 2012 Cochrane meta-analysis has continued to find no clear or convincing evidence of superiority on any measure apart from leaving the study early (Jones et al., 2018).

In the wake of a 'viewpoint' article examining whether it is right to continue to regard CBT as a gold standard for depression and anxiety (Leichsenring and Steinert, 2017), we consider the current status of this form of therapy in schizophrenia and the related psychotic disorders that are typically included in trials (schizoaffective disorder, delusional disorder, and psychosis not otherwise specified). We draw on what can legitimately be regarded as the two best sources of evidence, namely meta-analyses and large, well-conducted individual trials.

\section{Is CBT effective in high-quality trials?}

Figure 1 shows pooled ESs for positive symptoms, the class of symptoms that CBT was originally developed to treat, in meta-analyses carried out since 2001. A fall over time is evident, with meta-analyses carried out in the last 5 years all finding pooled ESs in the small range (0.1-0.3). It seems likely that this reflects the larger sample sizes and increased attention to methodological factors that have tended to characterize more recent trials.

In our 2014 meta-analysis (Jauhar et al., 2014), we found a significant moderating effect of blinding: the pooled ES reduced from 0.33 to 0.15 (95\% CI $0.03-0.27)$ in 20 trials of overall symptoms, and fell from 0.25 to non-significant levels [0.08 (95\% CI -0.03-0.18) in 20 trials of positive symptoms (Jauhar et al., 2014)]. Bighelli et al. (2018) had more nuanced results in their network meta-analysis: they found the small but significant ES for CBT was maintained in blind studies when compared against TAU $(0.27,95 \%$ CI $0.13-0.41)$ but not against inactive control interventions $(0.14,95 \%$ CI $-0.09-0.37)$.

Of course, blinding is not the only measure that contributes to trial quality - others include randomization (especially allocation concealment, the taking of steps to ensure that the randomization code cannot be broken, typically by use of remote allocation) (Schulz and Grimes, 2002a), and the use of measures to deal with incomplete outcome data (Schulz and Grimes, 2002b). In our meta-analysis (Jauhar et al., 2014), we addressed this broader issue by pooling data from studies that were rated as being at low risk of bias from all three of these factors. ESs for overall and positive symptoms were small and non-significant in these studies [overall symptoms: 0.15 (95\% CI -0.01-0.32), eight studies; positive symptoms: 0.10 (95\% CI -0.09-0.28), nine studies] (Jauhar et al., 2014, p. 22014). However, pooling data only from trials deemed to be at low risk of bias not only severely restricts the study base but also depends on information given by authors about their methodology, which may be 
Fig. 1. Pooled effect sizes for CBT against positive schizophrenic symptoms in meta-analyses from 2001 (all - all controls; TAU treatment as usual; conint - control psychological interventions).

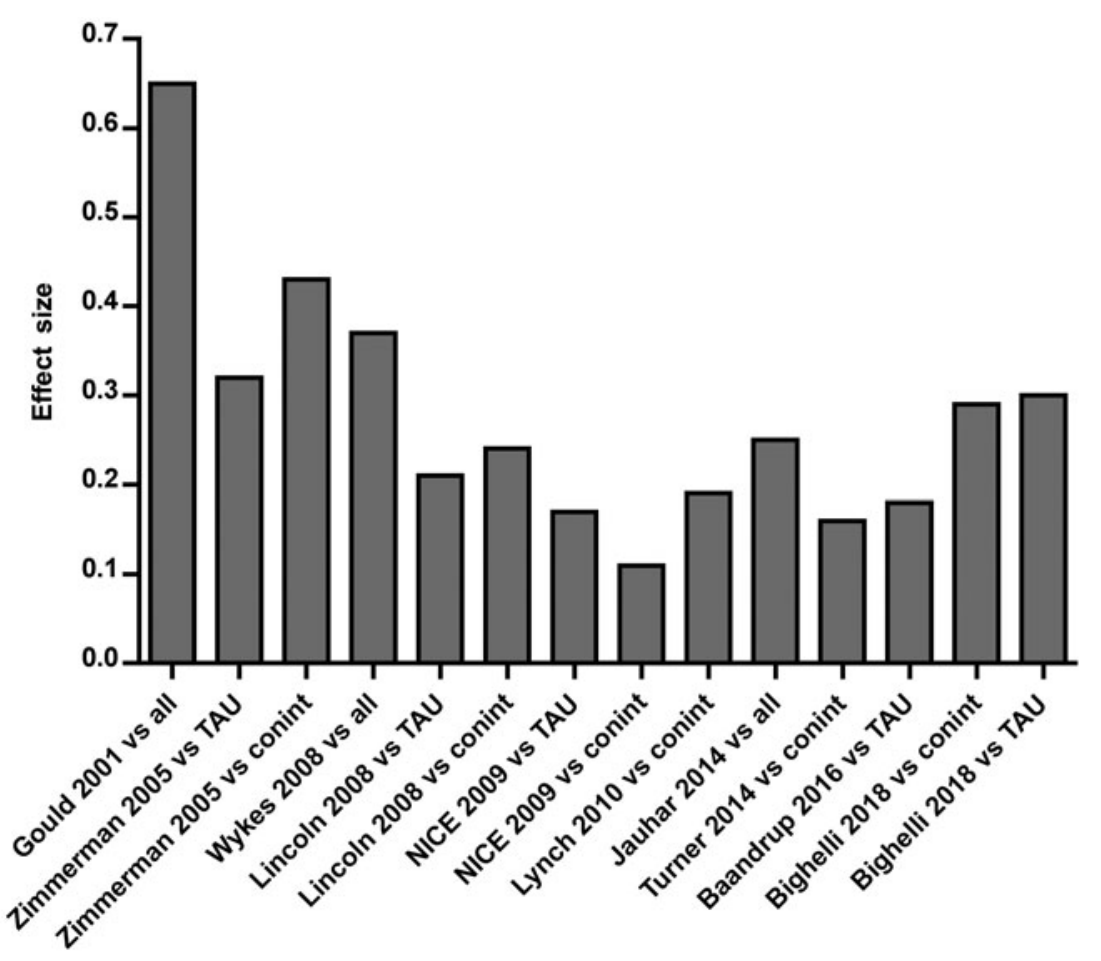

scanty, especially in older studies. In these circumstances, findings from a very large, methodologically rigorous, 'definitive' trial would be of considerable interest.

One such trial, the POSITIVE trial of Klingberg et al. (2010), comes close to meeting these requirements. Begun in 2007, this aimed to recruit 330 people diagnosed with schizophrenia, schizoaffective disorder or delusional disorder, who were randomized to 9 months of CBT or an intervention designed to control for nonspecific elements of therapeutic contact, supportive therapy. Randomization and blinding were state-of-the-art and analysis was planned to be by intention-to-treat. Publication of this trial has been delayed [for details see (see Coyne: http://blogs.plos. org/mindthebrain/2016/02/23/effect-of-a-missing-clinical-trial-onwhat-we-think-about-cognitive-behavior-therapy)], but is now anticipated to appear in the near future.

\section{Is CBT effective against particular symptoms?}

van der Gaag et al. (2014) meta-analyzed 11 studies that separately examined outcomes for delusions and hallucinations, and which were also selected on the basis that they used individually tailored, case formulation-based CBT. The findings for delusions were not greatly different from those of our 2014 meta-analysis (Jauhar et al., 2014) for positive symptoms: the pooled ES was 0.36 in nine studies, reducing to 0.24 in six blind studies. However, the ES was noticeably larger for hallucinations, being 0.44 in 11 studies and, at 0.46 , this value was maintained in eight blind studies.

The same year saw publication of the COMMAND trial of Birchwood et al. (2014), a large $(n=197)$, 9-month trial of hallucination-adapted CBT compared with TAU. This employed randomization by remote allocation, used multiple measures to prevent unblinding of assessors, and analysis was by intention-to-treat. The primary outcome was harmful compliance with voices: on this measure the CBT group showed significant superiority, although this was not seen at the end of treatment [odds ratio (OR) $0.74,95 \%$ CI 0.40-1.39] but became apparent at 18 -month follow-up (OR 0.45, 95\% CI 0.23-0.88). No significant effect on overall hallucinations severity was seen, however, at either time point, or on individual hallucination variables such as distress or frequency.

It seems increasingly clear that CBT is ineffective against negative symptoms. Velthorst et al. (2015) found no significant effect in 28 trials of negative symptoms as a secondary outcome [ES 0.09 ( $95 \%$ CI -0.03 to 0.21$)$ ], nor in two trials where negative symptoms were the primary outcome [ES 0.16 (95\% CI -0.10-0.41)]. One of these latter two trials (Klingberg et al., 2011) was large $(n=198)$, employed randomization by remote allocation, blinding, and analysis by intention-to-treat. Importantly, the form of CBT used was also adapted to specifically target negative symptoms (Klingberg et al., 2011).

\section{CBT for relapse}

NICE found an effect of CBT on reducing hospitalization compared with TAU in one of the three meta-analyses examining this outcome carried out for the 2009 guideline (see: National Collaborating Centre for Mental Health: www.nccmh.org.uk). On the other hand, there was no evidence of effectiveness against relapse, when compared with either standard care [relative risk (RR) 0.85 (95\% CI $0.50-1.41)$, three trials] or with other active treatments [RR 1.05 (CI 0.85-1.30), four trials].

Neither of these two latter meta-analyses included the large $(n=218)$ trial of Garety et al. (2008) of CBT $v$. TAU. This examined relapse rates based on evidence of re-emergence of or significant deterioration in positive psychotic symptoms lasting at least 2 weeks. The trial employed blinding, randomization by remote allocation, and analysis by intention-to-treat. Its results were negative, leading the authors to conclude generic CBT for psychosis was not indicated for relapse prevention in patients recovering from a recent episode of psychosis. 


\section{Is CBT more effective in treatment-resistant patients?}

Burns et al. (2014) meta-analyzed randomized trials of CBT in medication-resistant patients, finding apparently substantial end of treatment ESs of 0.52 (95\% CI $0.35-0.70)$ in 12 studies of overall symptoms and 0.47 (95\% CI $0.27-0.67)$ in nine studies of positive symptoms. However, they used an unconventional measure of ES (difference in change scores divided by end of treatment standard deviation) which does not permit their results to be quantified in terms of the widely used rule of thumb of small $(0.1-0.3)$, medium (0.4-0.7), and large (0.8+). Recalculation of the pooled ES for their nine studies of positive symptoms using a conventional metric (Hedges' $g$ ) gives an approximate result (values had to be estimated for one study) of 0.31 (95\% CI 0.12-0.50) falling to 0.26 (95\% CI 0.04-0.47) in seven blind studies - not markedly different from the values found in our meta-analysis of the same year (Jauhar et al., 2014).

The recently published FOCUS trial (Morrison et al., 2018) examined the effectiveness of CBT over a period of 9 months (plus later booster sessions) $v$. TAU in 487 patients with clozapine-resistant schizophrenia. As well as being very large, this trial was well-conducted, employed randomization by remote allocation and assessor blinding (with measures taken to address accidental breaking), and analysis was by means of a modern equivalent of intention-to-treat (White et al., 2011). No significant effect was seen at the primary endpoint of 21 months (ES 0.06 ), but a small effect (ES 0.16) was noted at the end of the 9-month treatment period.

\section{CBT: not a 'quasi-neuroleptic'?}

More than a decade ago, Birchwood and Trower (2006) argued that treating CBT as a 'quasi-neuroleptic' is inappropriate and that the intervention is more likely to have a distinctive profile of effects that is complementary to drug treatment rather than substituting for it. Such a view finds echoes in UK clinical guidelines: NICE (2009) emphasized not only effects on psychotic symptoms but also reduction of distress associated with symptoms, promotion of social and educational recovery, and reduction of depression and social anxiety. Similarly, SIGN (2013) stated: 'The aim [of CBT] is to help the individual normalize and make sense of their psychotic experiences, and to reduce the associated distress and impact on functioning'.

Laws et al. (2018) recently meta-analyzed these relatively understudied outcomes. In 27 trials that examined the effects of CBT on functioning, the pooled ES was small at end-of-trial [ES 0.25 (95\% CI 0.14-0.33)], although this became non-significant at follow-up [16 trials, ES 0.10 (95\% CI -0.17-0.26)]. A small-to-medium benefit on distress was found at end-of-treatment in eight trials [ES 0.37 (95\% CI 0.05-0.69)], which became non-significant when adjusted for possible publication bias [ES 0.18 (95\% CI -0.12-0.48)]. There was no evidence of an effect on quality of life in 10 trials [ES 0.04 (95\% CI -0.12-0.19)].

Freeman et al. (2015) examined another non-core outcome, worry secondary to delusions, in a large trial. One hundred and fifty patients with schizophrenia, schizoaffective psychosis, or delusional disorder who scored significantly on a worry questionnaire were randomly assigned to 8 weeks of either worry-directed CBT or TAU. At both end of treatment and 24 weeks follow-up the CBT group showed significantly reduced worry scores (ES estimate common to both outcome points 0.47 ). This trial was generally methodologically robust, but it should be noted that worry was measured on a self-report scale and so could not be considered to have been evaluated under blind conditions.

\section{Can CBT prevent transition to psychosis?}

Hutton and Taylor (2014) meta-analyzed six trials using CBT in individuals at risk of developing psychosis, and found evidence that it was effective in reducing the transition rate at 6,12 , and 18 months [6 months: RR 0.47 (95\% CI 0.27-0.82), six studies; 12 months: RR 0.45 (95\% CI 0.28-0.73), six studies; 18-24 months: RR 0.41 (95\% CI 0.23-0.72), four studies]. This meta-analysis included two large, well-conducted multicenter trials ( $n=288$, Morrison et al., 2012 and $n=206$, van der Gaag et al., 2012); the former failed to find a significant difference in transition frequency but the latter found a significant (55\%) reduction.

Interpretation of the findings in this area is complicated by a subsequent network meta-analysis that examined the effect of CBT and a range of other interventions (Davies et al., 2018). This found no significant effect of CBT on transition compared with standard clinical management. This meta-analysis excluded one of the two multicenter trials included by Hutton and Taylor (2014) (van der Gaag et al., 2012), on the grounds that it used CBT enhanced with psychoeducation and metacognitive strategies. It also included two new studies: one of these was a relatively small trial $(n=57)$ (Stain et al., 2016) which found three transitions in the CBT group but none in the control group, and the other was the as-yet unpublished PREVENT trial (Bechdolf et al., 2011) which randomized 216 individuals to CBT, antipsychotic treatment, or standard clinical management (plus placebo). A preliminary report of this study (Bechdolf et al., 2017) found a smaller number of transitions in the CBT group compared with clinical management (19.2\% v. 30.0\%), but the difference did not reach statistical significance.

\section{Conclusions}

CBT was originally introduced to treat the positive symptoms of schizophrenia, but its effect on these, according to convergent meta-analytic evidence, is small. A stronger effect on hallucinations remains a possibility, but this has to be treated with caution given that it is based on a meta-analysis of a relatively small number of studies (van der Gaag et al., 2014), and a subsequent large trial found no effect on voice frequency and distress (Birchwood et al., 2014). A considerable body of trials now makes it clear that CBT is ineffective against negative symptoms, either in generic or specially adapted forms. CBT, on present evidence, does not prevent relapse.

Where CBT may hold more promise is in areas of symptomatology not specifically targeted by antipsychotic drugs. Relatively large effects have been reported in large, well-conducted trials for worry related to delusions and harmful compliance with auditory hallucinations. The meta-analytic database on such symptoms, however, is small and not particularly encouraging as it stands.

The question of whether CBT is useful in preventing transition to psychosis in high-risk individuals currently hangs in the balance. The disagreement between the meta-analysis of Hutton and Taylor (2014) and the network meta-analysis of Davies et al. (2018) is clearly due to methodological factors, specifically study inclusion. The equivocal findings of the PREVENT trial - of a relatively substantial but statistically non-significant reduction in 
transition rate - will require integration into further meta-analyses for interpretation, and even then the conclusion may not be decisive.

Author ORCIDs. (D) S Jauhar, 0000-0002-3878-3659.

Conflict of interest. None.

\section{References}

Bechdolf A, Muller H, Stutzer H, Wagner M, Maier W, Lautenschlager M, Heinz A, de Millas W, Janssen B, Gaebel W, Michel TM, Schneider F, Lambert M, Naber D, Brune M, Kruger-Ozgurdal S, Wobrock T, Riedel M, Klosterkotter J and group Ps (2011). Rationale and baseline characteristics of prevent: A second-generation intervention trial in subjects at-risk (prodromal) of developing first-episode psychosis evaluating cognitive behavior therapy, aripiprazole, and placebo for the prevention of psychosis. Schizophrenia Bulletin 37 Suppl 2, S111-S121.

Bechdolf A, Müller H, Stützer H, Lambert M, Karow A, Zink M, Lautenschlager M, Heinz A, de Millas W, Janssen B, Gaebel W, Schneider F, Juckel G, Krüger-Özgürdal S, Wobrock T, Wagner M, Maier W and Klosterkötter J (2017). 108. PREVENT: a randomized controlled trial for the prevention of first-episode psychosis comparing cognitive-behavior therapy (CBT), clinical management, and aripiprazole combined and clinical management and placebo combined. Schizophrenia Bulletin 43, S56-S57.

Bighelli I, Salanti G, Huhn M, Schneider-Thoma J, Krause M, Reitmeir C, Wallis S, Schwermann F, Pitschel-Walz G, Barbui C, Furukawa TA and Leucht S (2018). Psychological interventions to reduce positive symptoms in schizophrenia: systematic review and network meta-analysis. World Psychiatry 17, 316-329.

Birchwood M and Trower P (2006). The future of cognitive-behavioural therapy for psychosis: not a quasi-neuroleptic. The British Journal of Psychiatry 188, $107-108$.

Birchwood M, Michail M, Meaden A, Tarrier N, Lewis S, Wykes T, Davies L, Dunn G and Peters E (2014). Cognitive behaviour therapy to prevent harmful compliance with command hallucinations (COMMAND): a randomised controlled trial. The Lancet Psychiatry 1, 23-33.

Burns AMN, Erickson DH and Brenner CA (2014). Cognitive-behavioral therapy for medication-resistant psychosis: a meta-analytic review. Psychiatric Services 65, 874-880.

Davies C, Cipriani A, Ioannidis JPA, Radua J, Stahl D, Provenzani U, McGuire P and Fusar-Poli P (2018). Lack of evidence to favor specific preventive interventions in psychosis: a network meta-analysis. World Psychiatry 17, 196-209.

Freeman D, Dunn G, Startup H, Pugh K, Cordwell J, Mander H, Černis E, Wingham G, Shirvell K and Kingdon D (2015). Effects of cognitive behaviour therapy for worry on persecutory delusions in patients with psychosis (WIT): a parallel, single-blind, randomised controlled trial with a mediation analysis. The Lancet Psychiatry 2, 305-313.

Garety PA, Fowler DG, Freeman D, Bebbington P, Dunn G and Kuipers E (2008). Cognitive-behavioural therapy and family intervention for relapse prevention and symptom reduction in psychosis: randomised controlled trial. The British Journal of Psychiatry 192, 412-423.

Hutton P and Taylor PJ (2014). Cognitive behavioural therapy for psychosis prevention: a systematic review and meta-analysis. Psychological Medicine 44, 449-468.

Jauhar S, McKenna PJ, Radua J, Fung E, Salvador R and Laws KR (2014). Cognitive-behavioural therapy for the symptoms of schizophrenia: systematic review and meta-analysis with examination of potential bias. The British Journal of Psychiatry 204, 20-29.

Jones C, Hacker D, Cormac I, Meaden A and Irving CB (2012). Cognitive behaviour therapy versus other psychosocial treatments for schizophrenia. The Cochrane Database of Systematic Reviews 4, CD008712.

Jones C, Hacker D, Meaden A, Cormac I, Irving CB, Xia J, Zhao S, Shi C and Chen J (2018). Cognitive behavioural therapy plus standard care versus standard care plus other psychosocial treatments for people with schizophrenia. The Cochrane Database of Systematic Reviews 11, CD008712.

Klingberg S, Wittorf A, Meisner C, Wölwer W, Wiedemann G, Herrlich J, Bechdolf A, Müller BW, Sartory G, Wagner M, Kircher T, König H-H, Engel C and Buchkremer G (2010). Cognitive behavioural therapy versus supportive therapy for persistent positive symptoms in psychotic disorders: the POSITIVE study, a multicenter, prospective, single-blind, randomised controlled clinical trial. Trials 11, 123.

Klingberg S, Wölwer W, Engel C, Wittorf A, Herrlich J, Meisner C, Buchkremer G and Wiedemann G (2011). Negative symptoms of schizophrenia as primary target of cognitive behavioral therapy: results of the randomized clinical TONES study. Schizophrenia Bulletin 37(Suppl. 2), S98-110.

Laws KR, Darlington N, Kondel TK, McKenna PJ and Jauhar S (2018). Cognitive behavioural therapy for schizophrenia - outcomes for functioning, distress and quality of life: a meta-analysis. BMC psychology 6, 32 .

Leichsenring F and Steinert C (2017). Is cognitive behavioral therapy the gold standard for psychotherapy?: the need for plurality in treatment and research. JAMA 318, 1323-1324.

Morrison AP, French P, Stewart SL, Birchwood M, Fowler D, Gumley AI, Jones PB, Bentall RP, Lewis SW, Murray GK, Patterson P, Brunet K, Conroy J, Parker S, Reilly T, Byrne R, Davies LM and Dunn G (2012). Early detection and intervention evaluation for people at risk of psychosis: Multisite randomised controlled trial. British Medical Journal 344, e2233.

Morrison AP, Pyle M, Gumley A, Schwannauer M, Turkington D, MacLennan G, Norrie J, Hudson J, Bowe SE, French P, Byrne R, Syrett S, Dudley R, McLeod HJ, Griffiths H, Barnes TRE, Davies L and Kingdon D, FOCUS trial group (2018). Cognitive behavioural therapy in clozapine-resistant schizophrenia (FOCUS): an assessor-blinded, randomised controlled trial. The Lancet Psychiatry 5, 633-643.

NICE (2009). Schizophrenia: Core interventions in the treatment and management of schizophrenia in adults in primary and secondary care (update). London: National Institute of Clinical Excellence.

NICE (2014). Psychosis and schizophrenia in adults: Treatment and management. National clinical guideline number 178. London: National Collaborating Centre for Mental Health/National Institute for Health and Care Excellence.

Rathod S, Phiri P and Kingdon D (2010). Cognitive behavioral therapy for schizophrenia. The Psychiatric Clinics of North America 33, 527-536.

Schulz KF and Grimes DA (2002a). Allocation concealment in randomised trials: defending against deciphering. The Lancet 359, 614-618.

Schulz KF and Grimes DA (2002b). Sample size slippages in randomised trials: exclusions and the lost and wayward. The Lancet 359, 781-785.

SIGN (2013). 131 Management of schizophrenia.

Stain HJ, Bucci S, Baker AL, Carr V, Emsley R, Halpin S, Lewin T, Schall U, Clarke V, Crittenden K and Startup M (2016). A randomised controlled trial of cognitive behaviour therapy versus non-directive reflective listening for young people at ultra high risk of developing psychosis: the detection and evaluation of psychological therapy (DEPTh) trial. Schizophrenia Research 176, 212-219.

van der Gaag M, Nieman DH, Rietdijk J, Dragt S, Ising HK, Klaassen RMC, Koeter M, Cuijpers P, Wunderink L and Linszen DH (2012). Cognitive behavioral therapy for subjects at ultrahigh risk for developing psychosis: a randomized controlled clinical trial. Schizophrenia Bulletin 38, 1180-1188.

van der Gaag M, Valmaggia LR and Smit F (2014a). The effects of individually tailored formulation-based cognitive behavioural therapy in auditory hallucinations and delusions: a meta-analysis. Schizophrenia Research 156, 30-37.

Velthorst E, Koeter M, van der Gaag M, Nieman DH, Fett A-KJ, Smit F, Staring ABP, Meijer C and de Haan L (2015). Adapted cognitivebehavioural therapy required for targeting negative symptoms in schizophrenia: meta-analysis and meta-regression. Psychological Medicine 45, 453-465.

White IR, Horton NJ, Carpenter J and Pocock SJ (2011). Strategy for intention to treat analysis in randomised trials with missing outcome data. BMJ 342, $\mathrm{d} 40$. 